\title{
VARIATION IN THE BANDWIDTHS OF PATTERN QUALITY \\ PARAMETERS AND MAXIMUM EMBEDDED IMPEDANCE AMONG THE SOLUTIONS TO SHAPED BEAM SYNTHESIS PROBLEMS FOR COLLINEAR DIPOLE ARRAYS
}

\author{
J. C. Brégains (Student Member IEEE), F. Ares (Senior Member IEEE), \\ and E. Moreno (Senior Member IEEE)
}

Radiating Systems Group, Department of Applied Physics, 15782 Campus Sur, Univ. of Santiago de Compostela (Spain)

Emails: (Ares)faares@usc.es - (Brégains)fajulio@usc.es

\begin{abstract}
We investigated variation in the bandwidths of ripple, side lobe level, $-3 \mathrm{~dB}$ beam width and maximum element embedded (active) impedance $\left(\left|\mathrm{Z}^{\mathrm{e}}\right|_{\max }\right)$ among the multiple collinear dipole arrays radiating a given flat-topped beam pattern. The main finding was that ripple and side lobe level bandwidths are much greater than when the dipoles are parallel.
\end{abstract}

\section{INTRODUCTION}

As is well known, the problem of synthesizing a linear antenna array radiating a given shaped beam pattern has $2^{\mathrm{Q}}$ solutions, where $\mathrm{Q}$ is the number of nulls of the corresponding sum pattern that have been filled to create the shaped beam [1]. In theory, this multiplicity should allow the choice of the solution offering best bandwidth as regards parameters measuring pattern quality (side lobe level, ripple, etc.) or antenna realizability (e.g. $\left|Z^{e}\right|_{\max }$, the maximum embedded impedance among the elements of the array). In the work described here we investigated this issue for arrays of collinear dipoles.

\section{THEORY}

We initially considered arrays of $\mathrm{N}=10$ and $\mathrm{N}=20$ collinear dipoles of length $\lambda_{\mathrm{D}} / 2$ and radius $0.004763 \lambda_{\mathrm{D}}$ [2] laid out along the $z$ axis with their centres $\lambda_{\mathrm{D}} / 2$ apart, where $\lambda_{\mathrm{D}}$ is the design wavelength. For each of these arrays we used the Orchard-Elliott method [1] to find a solution 
to the problem of synthesizing a symmetric flat-topped beam pattern with $\mathrm{Q}=2(\mathrm{~N}=10)$ or $\mathrm{Q}=$ $6(\mathrm{~N}=20)$ filled nulls, $\pm 0.5 \mathrm{~dB}$ of ripple in the shaped region, and a uniform side lobe level of $20 \mathrm{~dB}$. This method directly affords a set of complex roots $\exp \left(\mathrm{a}_{\mathrm{q}}+\mathrm{j} \mathrm{b}_{\mathrm{q}}\right)$ in the Schelkunoff equation $(1 \leq \mathrm{q} \leq \mathrm{Q})$, and the other $2^{\mathrm{Q}}-1=3(\mathrm{~N}=10)$ or $2^{\mathrm{Q}}-1=63(\mathrm{~N}=20)$ solutions for each array are then generated as usual by finding all possible combinations of signs of the $\mathrm{Q}=2$ or $\mathrm{Q}=$ 6 nonzero $a_{q}[1]$. For each solution we next calculated the mutual impedances $Z_{m n}$ and selfimpedances $\mathrm{Z}_{\mathrm{nn}}$ [3], and used them to obtain the input voltages $(1 \leq \mathrm{m}, \mathrm{n} \leq \mathrm{N})$

$$
\mathrm{V}_{\mathrm{m}}=\sum_{\mathrm{n}=1}^{\mathrm{N}} \mathrm{Z}_{\mathrm{mn}} \mathrm{I}_{\mathrm{n}}
$$

where the $I_{n}$ are the excitation currents constituting the solution in question.

Bandwidth was investigated for ripple, side lobe level (SLL), $-3 \mathrm{~dB}$ beam width, and $|\mathrm{Z}|_{\max }$. To this end, for each solution we fixed the $V_{n}$ obtained above and, for each of a sequence of frequencies differing by $\Delta f=f_{D} / 300$, we calculated first the corresponding $Z_{n m}$ [3]; then the corresponding $I_{n}$-by solving the system (1)- and the resulting power patterns (from which ripple, SLL and beamwidth values were obtained); and finally the embedded impedances $Z_{n}^{e}=V_{n} / I_{n}$. By this means, for each of the parameters of interest (ripple, etc.), we determined $f_{U}$ and $f_{L}$ (respectively the lowest frequency higher than $f_{D}$ and the highest frequency lower than $f_{D}$ at which the parameter was within a prespecified quantity $\delta$ of its value at $\mathrm{f}_{\mathrm{D}}$ ), and we calculated bandwidth as $\left(f_{U}-f_{L}\right) / f_{D}$, expressed as a percentage. The values of $\delta$ used were as follows: for $\left|Z^{e}\right|_{\max }$, $15 \%$ of the value of $\left|Z^{\mathrm{e}}\right|_{\max }$ at $\mathrm{f}_{\mathrm{D}}$; for beamwidth at $-3 \mathrm{~dB}, \pm 2^{\circ}$; for ripple, $\pm 0.5 \mathrm{~dB}$; and for SLL, $3 \mathrm{~dB}$ for levels higher than $20 \mathrm{~dB}$ and $\infty$ otherwise.

Once the 10- and 20-element arrays had been investigated as above, we investigated the same parameters for arrays with real symmetric current distributions that were required to radiate flattopped beams of approximately the same width as in the 10 - and 20 -element cases. For this purpose it was necessary [4] to increase the values of $\mathrm{N}$ and Q necessary to achieve patterns similar to those of the 10 - and 20 -element antennas being $\mathrm{N}=12$ and $\mathrm{Q}=4$ in the former case and $\mathrm{N}=$ 24 and $\mathrm{Q}=8$ in the latter. In these cases the solution for a given pattern is unique. 
Finally, all the above calculations were repeated for arrays with the same values of $\mathrm{N}$ and $\mathrm{Q}$ as before that had been shifted to $y=\lambda_{\mathrm{D}} / 4$ and endowed with an infinite ground plane at $y=0$. In this case, mutual and self-impedances were calculated as in [2-3]; the element factor was taken from the same source; and all patterns were calculated in the $y z$ plane.

\section{RESULTS}

The maximum and minimum bandwidth results obtained for arrays with no ground plane are listed in Table 1, where they are compared with results obtained in a similar study of arrays of parallel dipoles [5] (in the latter study, where the dipoles were oriented along $\mathrm{x}$ axis, all pattern parameters were calculated for the $y z$ plane, as in this work). The last two columns of Table 1 show whether the maximum- and minimum-bandwidth collinear array current distributions are real $(\mathrm{R})$ or complex $(\mathrm{C})$, and also whether they are symmetric $(\mathrm{S})$ or asymmetric $(\mathrm{A})$. The salient finding is that the ripple and SLL bandwidths of collinear arrays are much broader than those of the corresponding parallel arrays due to the lesser mutual coupling among the elements of the former. Beamwidth bandwidth is altered negligibly by the change in dipole orientation, and $\left|Z^{\mathrm{e}}\right|_{\max }$ bandwidth is slightly narrower for collinear than for parallel arrays (except in the case of the 24-element real symmetric array). Similar differences between parallel and collinear arrays were found in the presence of a ground plane (Table 2). For a given array type (collinear or parallel), the presence of a ground plane has relatively little effect, especially in the collinear case.

The excitation distributions affording the bandwidth extrema listed in Tables 1 and 2 may be obtained from the authors by email upon request.

\section{CONCLUSION}

The ripple and side lobe level bandwidths of linear arrays of $\lambda_{\mathrm{D}} / 2$-spaced dipoles radiating flat-topped beam patterns are much greater when the dipoles are collinear than when they are parallel. This is partly attributable to the mutual impedance between elements, which is 
stronger when the dipoles are parallel [6].

\section{ACKNOWLEDGEMENTS}

We thank Dr. R.C. Hansen for having suggested this research topic and for discussing its results at various stages.

This work was supported by the Spanish Ministry of Science and Technology under project TIC 2002-04084-C03-02.

\section{REFERENCES}

[1] H. J. Orchard, R. S. Elliott, and G. J. Stern, "Optimising The Synthesis Of Shaped Beam Antenna Patterns", IEE Proceedings, Vol. 132, pp. 63-69, 1985.

[2] R. S. Elliott, Antenna Theory and Design. Revised edition, John Wiley \& Sons, January 2003.

[3] R. C. Hansen "Formulation of Echelon Dipole Mutual Impedance for Computer", IEEE Trans. on Antennas and Propagat., Vol. 20, pp. 780-781, 1972.

[4] Y. U. Kim, and R. S. Elliott, "Shaped-Pattern Synthesis Using Pure Real Distributions", IEEE Trans. on Antennas and Propagat., Vol. 36, pp. 1645-1649, 1988.

[5] J. C. Brégains and F. Ares, "Variation in Bandwidth and in the Irregularity of Excitation Current, Input Voltage and Embedded Impedance Distributions among Solutions to Shaped Beam Synthesis Problems", Accepted for publication in IEEE Transactions on Antennas and Propagat.

[6] R. C. Hansen, "Phased Array Antennas", New York: John Wiley \& Sons, 1998. 


\section{LEGENDS FOR FIGURES AND TABLES}

\section{LEGENDS FOR THE TABLES}

Table 1. Bandwidths of ripple, side lobe level (SLL), $-3 \mathrm{~dB}$ beam width and $\left|\mathrm{Z}^{\mathrm{e}}\right|_{\max }$ of linear arrays of various sizes in the absence of a ground plane.

Table 2. As for Table 1 , but for arrays with a ground plane $\lambda_{\mathrm{D}} / 4$ away. 
TABLES

Table 1

\begin{tabular}{|c|c|c|c|c|c|c|c|}
\hline \multirow{2}{*}{$\begin{array}{c}\text { No. of } \\
\text { elements }\end{array}$} & \multirow{2}{*}{ Parameter } & \multicolumn{2}{|c|}{ Parallel } & \multicolumn{2}{|c|}{ Collinear } & \multicolumn{2}{|c|}{ Type } \\
\hline & & Max & Min & Max & Min & Max & Min \\
\hline \multirow{4}{*}{10} & BW Ripple \% & 13.00 & 11.33 & 151.00 & 68.67 & RA & $\mathrm{CS}$ \\
\hline & BW SLL \% & 57.17 & 54.17 & 188.83 & 149.83 & $\mathrm{CS}$ & $\mathrm{CS}$ \\
\hline & BW -3dB \% & 9.67 & 8.83 & 9.83 & 9.00 & RA & $\mathrm{CS}$ \\
\hline & BW $\left|\mathbf{Z}_{\mathrm{n}}^{\mathrm{e}}\right| \%$ & 14.67 & 10.50 & 10.33 & 8.50 & RA & $\mathrm{CS}$ \\
\hline \multirow{4}{*}{12} & BW Ripple \% & \multicolumn{2}{|c|}{10.33} & \multicolumn{2}{|c|}{138.17} & \multirow{4}{*}{\multicolumn{2}{|c|}{$\mathrm{RS}$}} \\
\hline & BW SLL \% & \multicolumn{2}{|c|}{42.50} & \multicolumn{2}{|c|}{167.67} & & \\
\hline & BW -3dB \% & \multicolumn{2}{|c|}{6.83} & \multicolumn{2}{|c|}{6.67} & & \\
\hline & BW $\left|Z_{\mathrm{n}}^{\mathrm{e}}\right| \%$ & \multicolumn{2}{|c|}{9.33} & \multicolumn{2}{|c|}{8.67} & & \\
\hline \multirow{4}{*}{20} & BW Ripple \% & 15.17 & 13.33 & 127.17 & 40.83 & RA & $\mathrm{CA}$ \\
\hline & BW SLL \% & 41.17 & 39.17 & 107.00 & 100.67 & $\mathrm{CS}$ & RA \\
\hline & BW -3dB \% & 8.83 & 7.67 & 8.33 & 7.50 & $\mathrm{CA}$ & $\mathrm{CA}$ \\
\hline & BW $\left|Z^{\mathrm{e}}\right| \begin{array}{l}\% \\
\end{array}$ & 15.83 & 5.17 & 15.33 & 1.67 & $\mathrm{CS}$ & RA \\
\hline \multirow{4}{*}{24} & BW Ripple \% & \multicolumn{2}{|c|}{8.67} & \multicolumn{2}{|c|}{127.83} & \multirow{4}{*}{\multicolumn{2}{|c|}{ RS }} \\
\hline & BW SLL \% & \multicolumn{2}{|c|}{30.33} & \multicolumn{2}{|c|}{121.83} & & \\
\hline & BW -3dB \% & \multicolumn{2}{|c|}{8.17} & \multicolumn{2}{|c|}{8.00} & & \\
\hline & BW $\left|\mathbf{Z}^{\mathrm{e}}\right| \begin{array}{l}\% \\
\end{array}$ & \multicolumn{2}{|c|}{4.33} & \multicolumn{2}{|c|}{10.50} & & \\
\hline
\end{tabular}

Table 2

\begin{tabular}{|c|c|c|c|c|c|c|c|}
\hline \multirow{2}{*}{$\begin{array}{c}\text { No. of } \\
\text { elements }\end{array}$} & \multirow{2}{*}{ Parameter } & \multicolumn{2}{|c|}{ Parallel } & \multicolumn{2}{|c|}{ Collinear } & \multicolumn{2}{|c|}{ Type } \\
\hline & & Max & Min & Max & Min & Max & Min \\
\hline \multirow{4}{*}{10} & BW Ripple \% & 15.00 & 14.00 & 152.00 & 62.67 & RA & $\mathrm{CS}$ \\
\hline & BW SLL \% & 70.83 & 69.83 & 191.17 & 191.00 & RA & $\mathrm{CS}$ \\
\hline & BW -3dB \% & 9.83 & 9.33 & 9.67 & 8.67 & RA & $\mathrm{CS}$ \\
\hline & BW $\left|Z^{\mathrm{e}}{ }_{\mathrm{n}}\right| \%$ & 14.83 & 9.33 & 9.67 & 7.83 & RA & $\mathrm{CS}$ \\
\hline \multirow{4}{*}{12} & BW Ripple \% & \multicolumn{2}{|c|}{11.50} & \multicolumn{2}{|c|}{136.00} & \multirow{4}{*}{\multicolumn{2}{|c|}{$\mathrm{RS}$}} \\
\hline & BW SLL \% & \multicolumn{2}{|c|}{67.50} & \multicolumn{2}{|c|}{190.33} & & \\
\hline & BW -3dB \% & \multicolumn{2}{|c|}{6.83} & \multicolumn{2}{|c|}{6.50} & & \\
\hline & BW $\left|Z^{\mathrm{e}}\right| \begin{array}{l} \\
\end{array}$ & \multicolumn{2}{|c|}{4.83} & \multicolumn{2}{|c|}{8.00} & & \\
\hline \multirow{4}{*}{20} & BW Ripple \% & 19.33 & 14.67 & 129.50 & 34.33 & RA & $\mathrm{CA}$ \\
\hline & BW SLL \% & 73.50 & 68.83 & 200.00 & 105.50 & $\mathrm{CA}$ & $\mathrm{CS}$ \\
\hline & BW -3dB \% & 8.50 & 7.83 & \begin{tabular}{|l|}
8.50 \\
\end{tabular} & 7.67 & RA & $\mathrm{CS}$ \\
\hline & BW $\left|Z^{\mathrm{e}}\right| \%$ & 15.67 & 2.00 & 13.83 & 1.67 & $\mathrm{CA}$ & RA \\
\hline \multirow{4}{*}{24} & BW Ripple \% & \multicolumn{2}{|c|}{10.17} & \multicolumn{2}{|c|}{129.50} & \multirow{4}{*}{\multicolumn{2}{|c|}{ RS }} \\
\hline & BW SLL \% & \multicolumn{2}{|c|}{73.67} & \multicolumn{2}{|c|}{200.00} & & \\
\hline & BW -3dB \% & \multicolumn{2}{|c|}{8.00} & \multicolumn{2}{|c|}{8.33} & & \\
\hline & BW $\left|Z^{e}\right| \%$ & \multicolumn{2}{|c|}{3.17} & \multicolumn{2}{|c|}{10.17} & & \\
\hline
\end{tabular}

